\title{
Variation in the use of cues to guide visual working memory
}

\author{
Matthew K. Robison $^{1} \cdot$ Nash Unsworth $^{1}$
}

Published online: 25 May 2017

(C) The Psychonomic Society, Inc. 2017

\begin{abstract}
Across two experiments we examined individual differences in the use of precues and retrocues to guide the selective encoding and maintenance of information in visual working memory (WM). In Experiment 1, we used spatial cues to indicate which item would be tested either before or after the presentation of a memory array. Regression analyses allowed us to separate variance due to visual WM capacity and attention control differences. In Experiment 2, we used categorical cues to indicate from which subset of items the tested item would be drawn, and we measured attention control with two independent tasks. Collectively, the results supported the idea that an element of individual differences in WM is the ability to flexibly allocate attention to encode only relevant information and subsequently select relevant information during maintenance stages.
\end{abstract}

\section{Keywords Visual working memory · Cueing · Attention} control

The relationship between attention control and working memory (WM) has been a major focus of research in cognitive psychology for decades. Research has shown that WM and attention control are distinct yet largely overlapping cognitive abilities (e.g., Engle \& Kane, 2004; Kane, Bleckley, Conway, \& Engle, 2001; Kane \& Engle, 2003). Latent variable analyses show that these two constructs share about $30-50 \%$ of their variance (Kane et al., 2016; McVay \& Kane, 2012; Redick et al., 2016; Robison, Gath, \& Unsworth, 2017; Unsworth,

Matthew K. Robison

mkr@uoregon.edu

1 Department of Psychology, 1227 University of Oregon, Eugene, OR 97403, USA
Fukuda, Awh, \& Vogel, 2014; Unsworth \& McMillan, 2014; Unsworth \& Spillers, 2010). Thus although they consistently covary, WM and attention control are not isomorphic. One element of many of these latent variable studies is that they have used attention control tasks that make little demand on WM other than maintenance of the task goal. The use of these tasks was intentional, as any shared variance between WM and attention control would be due to a domain-general executiveattention mechanism, rather than memory capacity per se. Therefore, these findings do not offer a specific glimpse into the allocation of attention to information being encoded into WM or being actively maintained within WM.

Other lines of research have specifically examined how individual differences in WM predict the control of attention within the scope of WM tasks. For example Vogel, McCollough, and Machizawa (2005) showed that individuals with greater visual WM capacity were more efficient at filtering out irrelevant items from WM. When presented with arrays containing irrelevant items, low-WM participants appeared to encode and maintain all items regardless of relevance, as reflected by contralateral delay activity (CDA; Vogel et al. 2005) and performance deficits for such trials. Low-WM individuals also seem less resistant to attention capture and take longer to recover from capture by irrelevant items (Fukuda \& Vogel, 2009, 2011). This line of research has subsequently argued that a crucial element of individual differences in WM is the ability to gate access to WM (Awh, Vogel, \& Oh, 2006; Cowan \& Morey, 2006; Fukuda \& Vogel, 2009, 2011; Unsworth \& Robison, 2016; Vogel et al., 2005).

However, other research argues that WM is primarily determined by individual differences in capacity per se - the amount of information an individual can encode and maintain in WM (i.e., scope of attention; Cowan et al., 2005). Recently Mall, Morey, Wolff, and Lehnert (2014) measured WM with complex span tasks, rather than visual change-detection tasks 
(Vogel et al., 2005). Their findings showed that low-WM individuals were equally likely, and in some cases less likely, to encode irrelevant information into WM compared to highWM individuals. From these findings, Mall et al. argue that variation in WM is not primarily due to differences in attention control, but rather to the amount of information an individual can maintain in WM. Therefore, the nature of the relationship between WM storage capacity (i.e., scope of attention) and the control of attention within WM tasks is still not entirely clear. Finally, some conceptualizations of WM and its relation with higher-order cognition argue that both attention control and storage capacity are important elements of WM (Chuderski \& Nęcka, 2012; Cowan, Fristoe, Elliott, Brunner, \& Saults, 2006; Engle, Tuhoski, Laughlin, \& Conway, 1999; Shipstead, Redick, Hicks, \& Engle, 2012; Unsworth et al., 2014; Unsworth, 2016). For example, Cowan et al. (2006) showed that both the scope of attention and the control of attention are important individual and developmental differences in terms of WM. Similarly, Shipstead et al. (2012) showed that the scope of attention and the control of attention are distinct yet related predictors of fluid intelligence. Further, Unsworth et al. (2014) showed that variation in attention control, storage capacity, and long-term memory fully mediated the relationship between complex WM span and fluid intelligence.

While many of the above-mentioned studies have focused on how individual differences in WM relate to the ability to selectively encode information, another important element of WM the ability to actively maintain information. For example, high-capacity participants are able to exert more attention when encoding items in WM then their low-capacity counterparts, as evidenced by differential patterns of pupil dilations during maintenance intervals (Unsworth \& Robison, 2015). Further, individual differences in CDA, an electrophysiological indicator of WM maintenance, correlate with other cognitive abilities like attention control, fluid intelligence, and longterm memory (Unsworth, Fukuda, Awh, \& Vogel, 2015). Therefore individual differences in the allocation of attention, both at encoding and during maintenance intervals, are important abilities that underlie effective cognition.

A separate yet related line of research has recently started using retrocueing paradigms, which have their roots in Sperling's (1960) partial reporting tasks, to examine the intricate interactions between attention and memory. Whereas filtering tasks (Fukuda \& Vogel, 2009; Fukuda \& Vogel, 2011; Mall et al., 2014; Unsworth \& Robison, 2016; Vogel et al., 2005) tell participants which items will be relevant ahead of time, retrocueing paradigms provide cues as to which items will be tested during maintenance intervals. Therefore, during the maintenance period, participants must flexibly adjust their attention to items within WM, rather than gate access at encoding. Retrocues are typically spatial in nature (Landman, Spekreijse, \& Lamme, 2003; Griffin \& Nobre,
2003), but can also be categorical (Astle, Scerif, Kuo, \& Nobre, 2009; Lepsien \& Nobre, 2007; Pertzov, Bays, Joseph, \& Husain, 2013). In general, the consistent finding is that retrocueing provides a benefit to participants as far as maintaining (or retrieving) the relevant item in working memory (Souza \& Oberauer, 2016).

The exact mechanism(s) by which retrocues operate on the contents of working memory are still not entirely clear. A wealth of recent research has tried to dissociate these various mechanisms (for review, see Souza \& Oberauer, 2016). At least six mechanisms have been proposed: protection from time-based decay (Matsukura et al., 2007; Pertzov et al., 2013), prioritization for comparison (e.g., Astle, Summerfield, Griffin, \& Nobre, 2012; Gözenman, Tanoue, Metoyer, \& Berryhill, 2014; Makovski et al., 2008; Matsukura et al., 2007; Pertzov et al., 2013), removal of irrelevant items (e.g., Williams, Hong, Kang, Carlisle, \& Woodman, 2013), attentional strengthening or refreshing (e.g., Chun \& Johnson, 2011), a retrieval head start (Souza, Rerko, \& Oberauer, 2016), and protection from perceptual interference (Makovski \& Jiang, 2007; Makovski et al., 2008; Matsukura et al., 2007; Pertzov et al., 2013; Slitge, Scholte, \& Lamme, 2008; Souza et al., 2016). In their rather comprehensive review of the existing literature on retrocues, Souza and Oberauer (2016) concluded that the majority of evidence supports the latter four hypotheses (i.e., removal, strengthening/refreshing, retrieval head start, and protection from interference). But the evidence for the first two possibilities (i.e., protection from time-based decay and prioritization for comparison) is rather mixed, at best. Because most of these mechanistic explanations are not mutually exclusive, they are difficult to disentangle experimentally. However, in the present study, our interest was not in the specific mechanism of the retrocue. Rather, the present study was designed to further define the relationship between WM and attention control by examining individual differences in the use of retrocues within visual WM tasks.

Several recent studies have started to investigate individual and developmental differences in the use of retrocues (Astle, Nobre, \& Scerif, 2012; Astle \& Scerif, 2011; Duarte et al., 2013; Gilchrist, Duarte, \& Verhaegen, 2016; Mok, Myers, Wallis, \& Nobre, 2016; Shimi, Kuo, Nobre, \& Scerif, 2014). These investigations have found that both children and adults benefit from retrocues, but children do not experience as much of a benefit as adults (Astle et al., 2012). Children show considerable variability in the use of retrocues, and this variability correlates with individual differences in visual WM capacity (Shimi et al., 2014). Among older adults, in order to be beneficial, cues may need to be about object-based features (e.g. shape, color) rather than spatial locations (Duarte et al., 2013; Gilchrist et al., 2016). There is meaningful variation in the use of retrocues among older adults, as well, and this covaries with neural oscillatory dynamics underlying WM maintenance 
(Mok et al., 2016). However, within the healthy young adult population, there has not been a systematic investigation of individual differences in WM and the use of retrocues. At least in one study retrocue task performance and spatial WM capacity were correlated, even after controlling for performance on neutral trials (Astle, Nobre, \& Scerif, 2012). Therefore, a primary purpose of the present study was to extend these findings to a larger sample of young adults. Additionally, Astle et al. (2012) measured retrocueing benefits with a spatial cue, and no study to date has measured individual differences in the use of a categorical retrocue and their relation to WM. Thus the use of retrocues within visual WM tasks provides an interesting paradigm within which we can test some fundamental issues about the nature of individual differences in attention control and WM.

The present study had three major aims. First, we wanted to investigate how individual differences in WM relate to the use of precues and retrocues to flexibly shift attention to items in visual WM, both at encoding and during maintenance. Second, we wanted to see how this relation generalized from the use of spatial cues to categorical cues. Third, and most broadly, we hoped to further define the role of attention control in determining individual differences in visual WM. To meet these aims, participants completed three complex span tasks to measure domain-general WM span. These tasks have been shown to have elements of short-term storage, attention control, and long-term memory retrieval (Unsworth \& Spillers, 2010; Unsworth et al., 2014). Participants then completed a visual WM task with three trial types: precue, neutral, and retrocue (Berryhill, Richmond, Shay, \& Olson, 2012). Precue trials were included to measure the allocation of attention at the level of encoding, whereas retrocue trials were included to measure attention allocation during maintenance. Neutral trials were included as our primary measure of visual WM capacity.

Table 1 shows a breakdown of specific hypotheses surrounding the relations among WM capacity, attention control, and the use of precues and retrocues, respectively. These hypotheses rely on regression, the logic of which closely follows Engle et al. (1999). Specifically, Engle et al. measured shortterm memory with simple span tasks, WM with complex span tasks, and fluid intelligence with matrix reasoning tasks.
Using structural equation modeling, Engle et al. argued that the shared variance between short-term memory and complex WM span was driven by individual differences in memory capacity, whereas the residual variance in complex WM span was attributable to attention control differences. In their model, Engle et al. showed that the shared variance between short-term memory and WM span (i.e., storage capacity) and the WM residual (i.e., attention control) both made significant independent contributions to fluid intelligence. So to isolate the variance in WM due to attention control, Engle et al. controlled for variation in short-term memory. Following that logic, we use regression to isolate shared and unique variance in cued trials attributable to neutral trials performance and WM span. Therefore, we operate under the following assumptions: (1) the shared variance between neutral trial performance and cued trial performance reflects abilities that are common to those two types of trials, and (2) any additional variance in cued trials explained by WM span reflects a form of attention control (i.e., selective encoding, selective maintenance) that is unique to cued trials. If, on the one hand, WM span explains no additional variance in cued trials over and above that explained by neutral trials, then there may be nothing unique about cued trials and their relationship with WM span. In other words, they make the same demands on WM capacity and attention control as neutral trials. If, on the other hand, there is a significant amount of shared variance between WM span and cued trials beyond the shared variance between neutral and cued trials, this would suggest that there is an element of individual differences in WM that involves the selective encoding and/or maintenance of relevant information. We do not argue that neutral trials are a process pure measure of visual WM capacity per se. But when entered into a simultaneous regression to predict cued trials, we argue that the shared variance between neutral trials and cued trials reflects shared capacity and control requirements across trial types, whereas WM span reflects additional control abilities that are further manifestations of individual differences in WM.

If WM span accounts for a significant portion of variance in precue trial performance after accounting for shared variance between neutral and cued trial performance, this would

Table 1 Theoretical interpretations of use of pre- and retrocues and predictions for the present study

\begin{tabular}{lc}
\hline Hypothesis & Prediction \\
\hline 1. Precue $=$ capacity + control & $\begin{array}{c}\text { Both neutral trial performance and WM span will account for significant portions of variance in } \\
\text { precue trial performance } \\
\text { All covariance between precue trial performance and WM span will be accounted for by neutral } \\
\text { trial performance }\end{array}$ \\
$\begin{array}{l}\text { 2. Precue }=\text { capacity } \\
\text { 3. Retrocue }=\text { capacity }+ \text { control } \\
\text { in retrocue trial performance }\end{array}$ \\
$\begin{array}{c}\text { All covariance between retrocue trial performance and WM span will be accounted for by neutral } \\
\text { trial performance }\end{array}$
\end{tabular}


indicate that selective encoding and visual WM capacity (the shared variance between neutral and precue trials) are both important elements of individual differences in WM (Hypothesis 1). Another possibility is that the shared variance between precue trial performance and WM span would be entirely due to the shared variance between neutral and cued trials (Hypothesis 2). We know that visual WM tasks correlate well with complex span WM tasks, even when there is no additional selective encoding or maintenance required (Shipstead, Harrison, \& Engle, 2015; Unsworth et al., 2014). So if precue and retrocue tasks draw upon capacity and control in a similar manner to uncued visual WM tasks, WM span will account for no independent variance. This hypothesis is most in line with the idea that individual differences in WM are largely important because of variation in storage capacity (Cowan et al., 2005; Mall et al., 2014). In other words, individuals are better at using precues primarily because they have a larger capacity to maintain items in visual WM.

With respect to retrocues, if there is a significant portion of shared variance between WM span and retrocue trial performance beyond the shared variance between neutral and retrocue trials, this would suggest both capacity and attention control are important for the selective maintenance of WM representations (Hypothesis 3). In addition to being able to maintain a larger number of items, highWM participants are also better able to flexibly allocate their attention to items within WM. However, if the WM span-retrocue relation is entirely accounted for by neutral trial performance, this would suggest that the use of retrocues is primarily due to the WM span's relation with capacity (Hypothesis 4). Individuals with a greater WM span can maintain more items, and therefore the retrocue will simply be more likely to point to an item or subset of items being actively maintained in visual WM.

\section{Experiment 1}

We used a task similar to that used by Berryhill et al. (2012) to examine individual differences in the use of spatial cues to guide attention toward items at encoding and during maintenance. The cues presented before the array allowed participants to select the relevant item at encoding, whereas the cues presented after the array forced participants to encode all items and subsequently select the relevant item during maintenance.

\section{Method}

\section{Participants and procedure}

A total of 179 participants were recruited through the undergraduate human subject pool at the University of Oregon. Two participants were excluded for performance at or near chance on the visual WM task, and one participant did not have operation span data because of a computer error, leaving a final sample of 176 participants. All participants first completed three complex span tasks followed by the visual WM task. The four tasks comprised about 45 min of a 2-hour session, during which participants completed long-term memory and reasoning tasks that were irrelevant to the present investigation, and the results of which have been reported elsewhere (Robison \& Unsworth, 2017a, b).

\section{Tasks}

Operation span In this task, participants solved a series of math operations while trying to remember a set of unrelated letters. Participants were required to solve a math operation, and after solving the operation, they were presented with a letter for $1 \mathrm{~s}$. Immediately after the letter was presented the next operation was presented. At recall participants were asked to recall letters from the current set in the correct order by clicking on the appropriate letters. For all of the span measures, items were scored as correct if the item was recalled correctly from the current list in the correct serial position. Participants were given practice on the operations and letter recall tasks only, as well as two practice lists of the complex, combined task. List length varied randomly from three to seven items, and there were two lists of each length for a total possible score of 50 . The score was total number of correctly recalled items in the correct serial position.

Symmetry span Participants recalled sequences of red squares within a matrix while performing a symmetryjudgment task. In the symmetry-judgment task, participants were shown an $8 \times 8$ matrix with some squares filled in black. Participants decided whether the design was symmetrical about its vertical axis. The pattern was symmetrical half of the time. Immediately after determining whether the pattern was symmetrical, participants were presented with a $4 \times 4$ matrix with one of the cells filled in red for $650 \mathrm{~ms}$. At recall, participants recalled the sequence of red-square locations by clicking on the cells of an empty matrix. Participants were given practice on the symmetry-judgment and square recall task as well as two practice lists of the combined task. List length varied randomly from two to five items, and there were two lists of each length for a total possible score of 28 . We used the same scoring procedure as we used in the operation span task.

Reading span While trying to remember an unrelated set of letters, participants were required to read a sentence and indicated whether or not it made sense. Half of the sentences made sense, while the other half did not. Nonsense sentences were created by changing one word in an otherwise normal 
sentence. After participants gave their response, they were presented with a letter for $1 \mathrm{~s}$. At recall, participants were asked to recall letters from the current set in the correct order by clicking on the appropriate letters. Participants were given practice on the sentence judgment task and the letter recall task, as well as two practice lists of the combined task. List length varied randomly from three to seven items, and there were two lists of each length for a total possible score of 50 . We used the same scoring procedure as we used in the operation span and symmetry span tasks.

Pre/retrocue task This task was modeled after that used by Berryhill et al. (2012). Participants completed a four-item change detection task with three types of trials: neutral, precue, and retrocue. Each trial started with a 1,200 ms fixation screen with a white cross centered on a black background. After a 200-ms blank screen, the precue appeared for $100 \mathrm{~ms}$. On neutral and retrocue trials, the precue was a white X. On precue trials, the cue was a white directional arrow pointing to one of the four locations. After a 400-ms blank screen, the memory array appeared for $300 \mathrm{~ms}$. After a 1,000-ms delay, the retrocue appeared and remained on-screen for $100 \mathrm{~ms}$. On neutral and precue trials, the retrocue was a white $\mathrm{X}$. On retrocue trials, the cue was a white directional arrow pointing to one of the four locations. Cues were $100 \%$ valid, and preand retrocues did not appear on the same trials. After another 400-ms delay, the tested item reappeared and remained onscreen until the participant made a same/different judgment about the color of the item. Participants made their response by pressing one of two keys labeled "S" and "D" for "same" and "different" (the F and J keys on the keyboard). Participants then pressed the space bar to start the next trial (Fig. 1). Participants completed 12 practice trials and 48 scored trials. Trials were presented in a random order (16 precue, 16 retrocue, and 16 neutral). The stimulus was equally likely to appear in each of the four locations. The color of the tested item changed on $50 \%$ of trials.

\section{Results}

Correlations and descriptive statistics for all measures are shown in Table 2. The measures showed good variability among participants, and values of skewness and kurtosis were within acceptable ranges (Kline, 2011). The dependent variable for the neutral, precue, and retrocue trials was accuracy. ${ }^{1}$ As the three complex span tasks showed acceptable internal consistency $(\alpha=0.72)$, we computed a composite WM span score for each participant using principal axis factoring and allowing the three tasks to load onto a single factor. The

\footnotetext{
${ }^{1}$ We used accuracy as the dependent variable for analyses. Using $k$ estimates or $d^{\prime}$ led to qualitatively similar sets of results, so we only report analyses on accuracy.
}

resulting factor loadings for operation span, symmetry span, and reading span were $.88, .52$, and .70 , respectively. This factor score is used in all subsequent analyses involving WM span.

A repeated-measures ANOVA on accuracy with trial-type (neutral, precue, retrocue) as a within-subjects factor revealed a main effect of trial type $(F(2,350)=192.13, p<0.001$, partial $\eta^{2}=0.52$ ). Bonferroni-corrected comparisons revealed that accuracy was significantly higher for precue trials compared to retrocue trials $(p<0.01)$, which had significantly higher accuracy than neutral trials $(p<0.01)$. Adding WM span as a covariate to the model did not reveal a significant WM span by trial-type interaction $(F<1)$. But there was a main effect of WM span such that individuals with greater WM span had higher accuracy overall $(F(1,174)=$ $6.39, p=0.01$, partial $\left.\eta^{2}=0.04\right)$. WM span did not significantly correlate with neutral trial accuracy, but did significantly correlate with precue and retrocue trial accuracy. However, the correlations between WM span and neutral accuracy, precue accuracy, and retrocue accuracy were not significantly different from one another ( $p s>.46)$.

Although the results of the ANCOVA suggest that the use of precues and retrocues is not a specific advantage for individuals with greater WM span, regression allowed us to separate shared and unique variance in precue and retrocue accuracy attributable to neutral trial accuracy and WM span. It could be the case that the relationship between WM span and precue and retrocue accuracy is entirely attributable to neutral trial accuracy (Table 1, Hypotheses 2 and 4). This would suggest that cued trials require no additional attention control beyond what is required during neutral trials. However, if WM span accounts for a significant portion of variance in precue and retrocue accuracy after controlling for neutral trial accuracy, this suggests that an element of individual differences in WM is the ability to flexibly allocate attention to items at both encoding and maintenance (Hypotheses 1 and 3). To test these possibilities, we ran stepwise regressions on precue and retrocue accuracy with neutral trial accuracy and WM span as predictors (Tables 3 and 4).

In Step 1 of the regression on precue accuracy (Table 3), we estimate the variance explained by neutral trial accuracy. Precue trials and neutral trials share a number of features, as they are part of the same task and are both visual changedetection tasks, so they require many overlapping mechanisms such as the allocation of attention at encoding, maintenance over a brief delay, and comparison to a test item. Indeed, about $3 \%$ of variance in precue trials was explained by neutral trial performance. The additional effect of adding WM span as a predictor in Step 2 did not reach traditional thresholds for significance $(p=.08)$. But it did suggest there may be something unique about precue trials that WM span is predicting (about $2 \%$ of variance). We argue that this variance is due to a selective-attention ability during the initial 


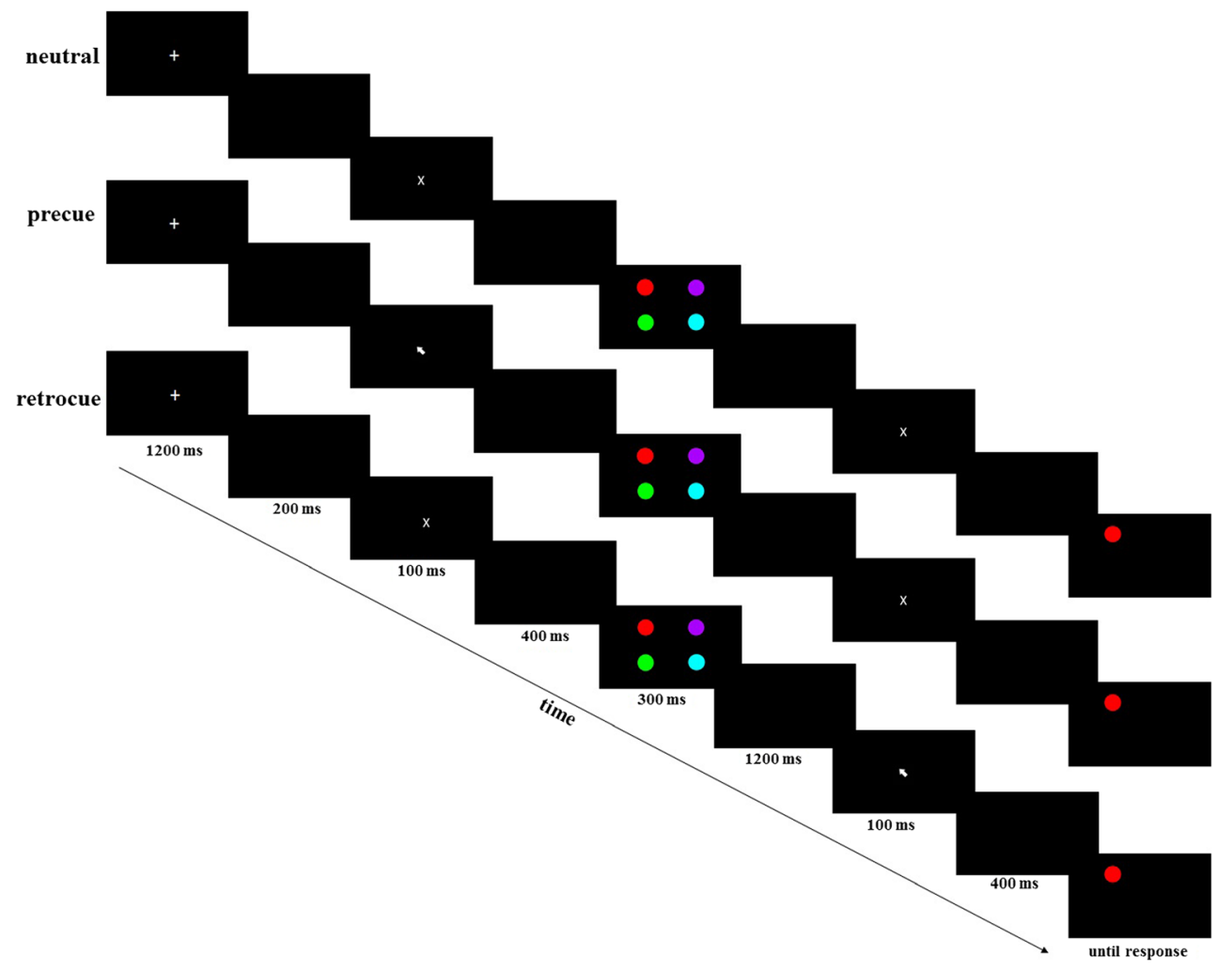

Fig. 1 Depiction of three trial types in Experiment 1

encoding period. Admittedly, the relationships are rather weak. But that is not entirely surprising, as the precues led to high average accuracy $(M=.94)$, and they effectively narrowed the set size down from four items to one. So if anything, it is a bit surprising that there was enough variability across participants to allow for these analyses. Collectively the results are most in line with Hypothesis 1 that both storage capacity and attention control, in this case the selective encoding of a specific item, are elements of individual differences in WM.

Table 2 Correlations and descriptive statistics for Experiment 1

\begin{tabular}{lllll}
\hline Measure & 1 & 2 & 3 & 4 \\
\hline 1. WM span & --- & & & \\
2. Neutral & .11 & --- & & \\
3. Precue & $.15^{+}$ & $.16^{*}$ & --- & \\
4. Retrocue & $.17^{*}$ & $.39^{*}$ & $.43^{*}$ & --- \\
Mean & .00 & .78 & .94 & .88 \\
SD & .90 & .11 & .06 & .08 \\
Skew & -.86 & -.72 & -1.68 & -.90 \\
Kurtosis & .93 & .25 & 3.85 & 1.14 \\
$\alpha$ & --- & .26 & .46 & .78 \\
\hline
\end{tabular}

Note: $N=176$

WM span average of standardized scores on operation span, symmetry span, and reading span, $S D$ standard deviation, $\alpha=$ Cronbach's alpha ${ }^{*} p<0.05,{ }^{+} p=.05$.
The second set of regressions analyzed retrocue accuracy (Table 4). Again, neutral trial accuracy accounted for most of the systematic variance in retrocue trial accuracy. Again, the effect of WM span was not quite significant $(p=.06)$. But after controlling for shared variance between neutral and retrocue trial performance, which we argue primarily reflects capacity differences, individual differences in WM span were still modestly predictive of retrocue trial performance. These results are most in line with Hypothesis 3 that both capacity and attention control are important elements of individual differences in WM. The control mechanism during retrocue trials is a bit different from the control required during precue trials. Rather than select an item at encoding, retrocues require (or at least encourage) participants to select the relevant item within

Table 3 Regression on precue trial accuracy for Experiment 1

\begin{tabular}{llllll}
\hline Predictor & & $\Delta R^{2}$ & $\beta$ & $t$ & $p$ \\
\hline Step 1 & & .03 & & & \\
& Neutral trial accuracy & & .16 & 2.17 & .03 \\
Step 2 & & .02 & & & \\
& Neutral trial accuracy & & .15 & 1.97 & .05 \\
& WM span & & .13 & 1.75 & .08 \\
Total $R^{2}$ & & .05 & & & \\
\hline
\end{tabular}

Note. $\mathrm{N}=176$

$\beta=$ standardized beta coefficient 
Table 4 Regression on retrocue trial accuracy for Experiment 1

\begin{tabular}{llllll}
\hline Predictor & & $\Delta R^{2}$ & $\beta$ & $t$ & $p$ \\
\hline Step 1 & & .15 & & & \\
& Neutral trial accuracy & & .39 & 5.55 & $<.001$ \\
Step 2 & & .02 & & & \\
& Neutral trial accuracy & & .37 & 5.39 & $<.001$ \\
& WM span & & .13 & 1.89 & .06 \\
Total $R^{2}$ & & .17 & & & \\
\hline
\end{tabular}

Note. $\mathrm{N}=176$

$\beta=$ standardized beta coefficient

WM. So this form of control is different, but also appears to be an important element of individual differences in WM.

\section{Discussion}

In Experiment 1 we used a cueing paradigm embedded within a visual WM task to test assumptions about the nature of capacity and attention control in determining individual differences in WM. Although the relationship between attention control and visual WM capacity has previously been documented, it is as yet unclear as to how exactly attention control is important for the selective encoding and maintenance of items in WM. Some have argued that individual differences in WM capacity are largely due to differences in the ability to gate access to WM (e.g., Vogel et al., 2005; Fukuda \& Vogel, 2009, 2011). Others have argued that differences are due more to the amount of information one can hold active in WM (Cowan et al., 2005; Mall et al., 2014). We used individual differences in the use of precues and retrocues to guide visual WM to test these ideas. The present findings suggest that both storage capacity limitations and the ability to control attention are fundamental aspects of WM performance (Cowan et al., 2006; Shipstead et al., 2012, Shipstead et al. 2015; Unsworth et al., 2014). On precue trials, the selective encoding of the relevant item required attention control. On retrocue trials, attention control was required during the selection of the cued item during the maintenance interval. Admittedly, the results were still a bit ambiguous as most of the relationships were rather weak. One of the problems was that participants were nearly at ceiling for precue trials, and performance on retrocue trials was also quite high, leaving little between-subject variance to examine. We addressed these weaknesses in Experiment 2 .

\section{Experiment 2}

Experiment 1 suggested that the use of spatial cues during a visual WM task related to individual differences in WM capacity and attention control. But some of the results were a bit ambiguous. To extend these findings, we used a different visual WM task, one that has traditionally been used to measure filtering abilities (Unsworth \& Robison, 2016; Vogel et al., 2005). To our knowledge, no study has used a categorical retrocue to investigate individual differences in WM and their relation to the use of such cues. Another crucial difference from Experiment 1 is that the task in Experiment 2 cued participants to a subset of items, rather than one specific item. A third difference is that the visual WM task in Experiment 2 has a larger set size. We designed the task this way to minimize the ceiling effects we observed in Experiment 1. Additionally, the design of the present study allowed us to test discrepant findings between accounts that favor a control perspective (Vogel et al., 2005) and those that favor a capacity perspective (Mall et al., 2014). Finally, we included two additional tasks to better account for differences in visual WM performance attributable to attention control. These tasks were chosen because they have previously been used in analyses of WM and attention control (Robison et al., 2017; Unsworth et al., 2014; Unsworth \& McMillan, 2014; Unsworth, McMillan, Brewer, \& Spillers, 2012; Unsworth, Brewer, \& Spillers, 2012) and load well onto an attention control factor. But importantly, they measure general goal-maintenance and sustained attention abilities, which are distinguishable from the sorts of attention control measured within visual WM tasks (e.g., filtering). Therefore, we can examine shared variance between visual WM-specific control differences (i.e., the use of precues and retrocues), and attention control differences more generally.

\section{Method}

\section{Participants and procedure}

A total of 189 participants completed the three complex span tasks and a visual WM task. The four tasks comprised the first half of a $2-h$ session. During the remaining time in the session, participants completed a long-term memory task that was irrelevant to the present investigation (Robison \& Unsworth, 2017). Ten participants were excluded from the final analyses because they had below-chance performance for at least one trial type. Four participants did not have data for one or both of the attention control tasks, and two participants did not have operation span scores due to computer errors, leaving a final sample of 171 participants. None of the participants in Experiment 2 participated in Experiment 1.

Tasks

Operation span. See Experiment 1. Symmetry span. See Experiment 1. Reading span. See Experiment 1. 
Psychomotor vigilance The psychomotor vigilance task (Dinges \& Powell, 1985) was used as the primary measure of sustained attention. Participants were presented with a row of zeros on screen and after a variable amount of time the zeros began to count up in $1-\mathrm{ms}$ intervals from $0 \mathrm{~ms}$. The participants' task was to press the spacebar as quickly as possible once the numbers started counting up. After pressing the space bar the response time was left on screen for $1 \mathrm{~s}$ to provide feedback to the participants. Interstimulus intervals were randomly distributed and ranged from $2 \mathrm{~s}$ to $10 \mathrm{~s}$. The entire task lasted for $10 \mathrm{~min}$ for each individual (roughly 75 total trials). The dependent variable was the mean reaction time for the slowest $20 \%$ of trials. ${ }^{2}$ Thought probes appeared randomly after $20 \%$ of trials.

Antisaccade In this task (Kane et al., 2001) participants were instructed to stare at a fixation point which was on-screen for a variable amount of time (200-2,200 ms). A white equals sign ("=") was then flashed either to the left or right of fixation (11.33 ${ }^{\circ}$ of visual angle) for $100 \mathrm{~ms}$, followed by a $50-\mathrm{ms}$ blank screen, then re-appearing for another $100 \mathrm{~ms}$. After a 50-ms blank interval, the target stimulus (a B, P, or R) appeared on-screen for $100 \mathrm{~ms}$. This was followed by masking stimuli (an $\mathrm{H}$ for $50 \mathrm{~ms}$ followed by an 8 which remained onscreen until a response was given). The participants' task was to identify the target letter by pressing a key for B, P, or R (the keys 4,5 , or 6 ) as quickly and accurately as possible. In the prosaccade condition the flashing cue (=) and the target appeared in the same location. In the antisaccade condition the target appeared in the opposite location as the flashing cue. Participants received, in order, ten practice trials to learn the response mapping, 15 trials of the prosaccade condition, and 60 trials of the antisaccade condition. The dependent variable was proportion correct on the antisaccade trials. Thought probes appeared randomly after ten antisaccade trials.

Filtering task Each trial began with a 1,200-ms fixation screen on which a black fixation cross was centered on a gray screen. Following a 200-ms blank screen delay, the precue appeared for $250 \mathrm{~ms}$. On precued trials, the cue was the word "RED" in size 18 red Courier New font or "BLUE" in blue font. On neutral trials, the screen remained blank for the precue period. After another 250-ms delay, the stimuli appeared and remained on-screen for $300 \mathrm{~ms}$. Stimuli were red- and blue-colored rectangles that appeared in random spatial locations on the screen with one of four orientations (vertical, horizontal, $45^{\circ}$ tilt to the right, $45^{\circ}$ tilt to the left). Three red items and three blue items appeared on every trial. After a

\footnotetext{
${ }^{2}$ Before computing each participant's mean for their $20 \%$ slowest trials, we trimmed extremely short and extremely long trials by eliminating any reaction times shorter than $150 \mathrm{~ms}$ or longer than $3,000 \mathrm{~ms}$. This procedure eliminated less than $1 \%$ of total trials.
}

250-ms delay, the retrocue appeared and remained on-screen for $250 \mathrm{~ms}$. On retrocue trials, the cue was the word "RED" in red font or "BLUE" in blue font. On neutral and precue trials the screen remained blank during the retrocue period. Cues were $100 \%$ valid. After another 500-ms delay, the items reappeared and one colored rectangle had a white dot on it (Fig. 2). Participants indicated whether the rectangle with the white dot was the same orientation or a different orientation than its previous presentation with one of two keys marked "S" or "D" for "same" and "different" (the F and J keys on the keyboard). The test array remained on the screen until the participant made a response. Participants initiated the next trial by pressing the spacebar. Participants completed six practice trials followed by 60 scored trials ( 20 precue, 20 neutral, 20 retrocue).

Thought probes Although they were not included as part of the present investigation, thought-sampling probes were including in the psychomotor vigilance and antisaccade tasks. Periodically, participants were prompted with a screen that said:

Please characterize your current conscious experience.

1) I am totally focused on the current task.

2) I am thinking about my performance on the task.

3) I am distracted by sights/sounds in my environment.

4) I am thinking about things unrelated to the task.

5) I am not very alert/my mind is blank.

Participants pressed the key that best corresponded to their current thoughts and proceeded with the task. Because they were not included as part of the present investigation, specifically, we did not analyze them in relation to the other dependent variables of interest (see Discussion).

\section{Results}

Correlations and descriptive statistics among the complex span and attention control measures are shown in Table 5. To create WM span and attention control factors, we used principal axis factoring to compute a factor score for each participant. These factor scores were used in all subsequent analyses involving WM span and attention control. Descriptive statistics for the factor scores and correlations with the three filtering task trial types are shown in Table 6.

A repeated-measures ANOVA with trial type (neutral, precue, retrocue) as a within-subjects factor revealed a main effect of trial type $(F(2,340)=118.86, p<.001$, partial $\left.\eta^{2}=.41\right)$. Bonferroni-corrected follow-up comparisons showed that accuracy was significantly higher on precue trials compared to retrocue trials $(p<.001)$, which were significantly more accurate than neutral trials $(p<.001)$. Adding the 


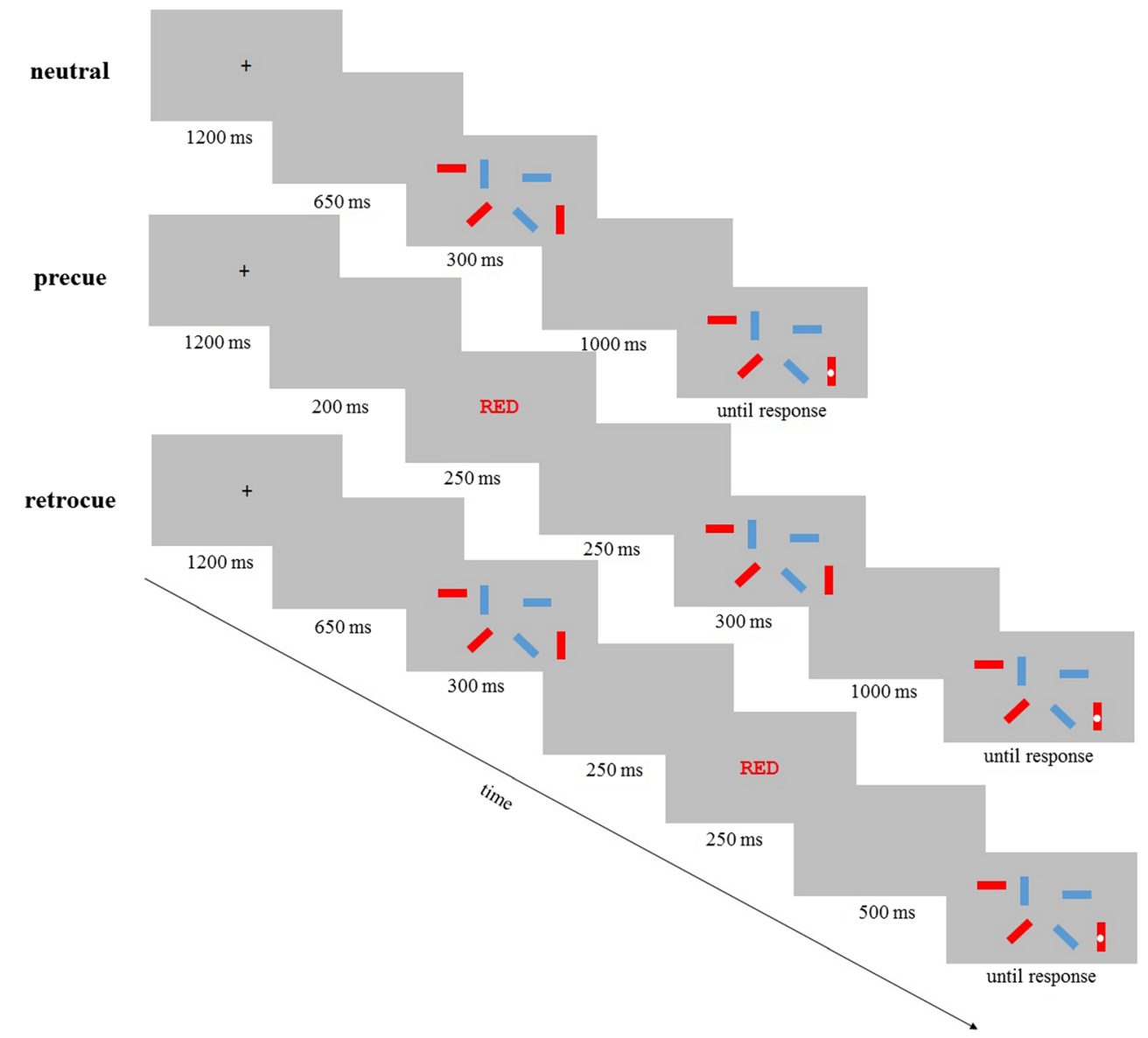

Fig. 2 Depiction of a neutral trial, a precue trial, and a retrocue trial in Experiment 2

WM span and attention control factors as covariates to the model revealed significant main effects of WM span $(F(1$, 168) $=11.55, p=.001$, partial $\left.\eta^{2}=.06\right)$ and attention control $\left(F(1,168)=14.17, p<.001\right.$, partial $\left.\eta^{2}=.08\right)$, such that individuals with greater WM span and attention

Table 5 Correlations and descriptive statistics for complex span and attention control tasks

\begin{tabular}{llllll}
\hline Measure & 1 & 2 & 3 & 4 & 5 \\
\hline 1. Operation span & --- & & & & \\
2. Symmetry span & $.42^{*}$ & --- & & & \\
3. Reading span & $.45^{*}$ & $.41^{*}$ & --- & & \\
4. Psychomotor vigilance & -.08 & $-.18^{*}$ & -.11 & --- & \\
5. Antisaccade & .13 & $.35^{*}$ & $.22^{*}$ & $-.31^{*}$ & --- \\
Mean & 37.87 & 19.96 & 36.46 & .49 & 523 \\
$S D$ & 7.25 & 4.65 & 8.07 & .14 & 165 \\
Skew & -.57 & -.47 & -.65 & 3.24 & .34 \\
Kurtosis & .002 & .17 & .27 & 14.25 & -.49 \\
$\alpha$ & .60 & .51 & .68 & .94 & .73 \\
\hline
\end{tabular}

Note. $N=171$

$S D$ standard deviation

$* p<.05$. control had higher accuracy overall. The trial-type $\times$ WM span interaction was not quite significant $(F(2,336)=$ 2.86, $p=.06$, partial $\left.\eta^{2}=.02\right)$. Attention control did not

Table 6 Correlations and descriptive statistics for working memory (WM) span and attention control factors and filtering

\begin{tabular}{|c|c|c|c|c|c|}
\hline Measure & 1 & 2 & 3 & 4 & 5 \\
\hline 1. WM span & --- & & & & \\
\hline 2. $\mathrm{AC}$ & $.28^{*}$ & --- & & & \\
\hline 3. Neutral & $.15^{+}$ & $.24 *$ & --- & & \\
\hline 4. Precue & $.36^{*}$ & $.36^{*}$ & $.31 *$ & --- & \\
\hline 5. Retrocue & $.22 *$ & $.19^{*}$ & $.32 *$ & $.51 *$ & --- \\
\hline Mean & .00 & .00 & .68 & .83 & .74 \\
\hline SD & .84 & .69 & .10 & .12 & .12 \\
\hline Skew & -.64 & -1.17 & .42 & -.76 & -.18 \\
\hline Kurtosis & .48 & 3.40 & .30 & .001 & -.69 \\
\hline$\alpha$ & --- & --- & .26 & .54 & .48 \\
\hline \multicolumn{6}{|c|}{ Note. $N=171$} \\
\hline \multicolumn{6}{|c|}{$\begin{array}{l}\text { WM span factor score derived from three complex span tasks, } A C \text { factor } \\
\text { score derived from psychomotor vigilance and antisaccade tasks, } S D \\
\text { standard deviation }\end{array}$} \\
\hline \multicolumn{6}{|c|}{$* p<0.05,{ }^{+} p=.05$} \\
\hline \multicolumn{6}{|c|}{$\alpha=$ reliability estimate } \\
\hline
\end{tabular}


interact with trial type $(F(2,336)=1.39, p=.25$, partial $\eta^{2}=.02$ ). WM span and attention control correlated significantly with all types of trials in the visual WM task. The small and non-significant WM span $\times$ trial-type interaction was driven by the fact that correlation between WM span and precue trials was significantly greater than the correlation between WM and neutral trials $(z=2.45, p=.01)$. The correlation between WM span and retrocue trials did not significantly differ from the correlation between WM span and neutral trials $(p=.42)$.

Similar to Experiment 1, we ran stepwise regressions on precue and retrocue trial accuracy to examine shared and unique variance accounted for by neutral trial accuracy, WM span, and attention control (Tables 7 and 8). In Step 1, we accounted for shared variance between neutral trial accuracy and precue trial accuracy. Neutral trial accuracy accounted for about $10 \%$ of variance in precue trials. Adding attention control and WM span in Step 2 accounted for an additional $15 \%$ of variance, and all three were significant predictors in the simultaneous model. Therefore, there is something unique about WM span and attention control in their ability to predict precue trials. This result is most in line with Hypothesis 1 in which both capacity and attention control are important for effective WM performance, especially when filtering of irrelevant items is required. Furthermore, even after controlling for attention control and neutral trial accuracy, WM span accounted for a significant portion of variance in precue trial performance. In the General discussion, we address some possibilities for why this might be the case.

In regard to retrocues, neutral trial accuracy accounted for about $8 \%$ of variance in Step 1. Adding WM span and attention control to the model in Step 2 accounted for an additional $4 \%$ of variance, but only WM span and neutral trial accuracy were significant predictors in the model. So after controlling for shared variance between neutral and retrocue trial accuracy, attention control no longer accounted for a significant portion of variance in retrocue accuracy. However, WM span did make a significant contribution. So some element of WM span, perhaps the

Table 7 Regression on precue trial accuracy for Experiment 2

\begin{tabular}{llllll}
\hline Predictor & & $\Delta R^{2}$ & $\beta$ & $t$ & $p$ \\
\hline Step 1 & & .10 & & & \\
& Neutral trial accuracy & & .31 & 4.24 & $<.001$ \\
Step 2 & & .15 & & & \\
& Neutral trial accuracy & & .22 & 3.09 & .002 \\
& WM Span & & .32 & 4.73 & $<.001$ \\
& Attention control & & .24 & 3.32 & .001 \\
Total $R^{2}$ & & .25 & & & \\
\hline
\end{tabular}

Note. $N=171$
Table 8 Regression on retrocue trial accuracy for Experiment 2

\begin{tabular}{llllll}
\hline Predictor & & $\Delta R^{2}$ & $\beta$ & $t$ & $p$ \\
\hline Step 1 & & .10 & & & \\
& Neutral trial accuracy & & .32 & 4.37 & $<.001$ \\
Step 2 & & .04 & & & \\
& Neutral trial accuracy & & .28 & 3.71 & $<.001$ \\
& WM span & & .16 & 2.10 & .04 \\
& Attention control & & .08 & 1.04 & .30 \\
Total $R^{2}$ & & .14 & & & \\
\hline
\end{tabular}

Note. $N=171$

ability to select and retrieve relevant items in memory (Unsworth \& Engle, 2007), is also important for the use of retrocues in WM. Although attention control was not a significant predictor of retrocue trial performance, it did significantly correlate with retrocue accuracy $(r=.19, p=.01)$. But it appears this covariance was shared with neutral trial accuracy and WM span.

\section{Discussion}

Experiment 2 largely replicated Experiment 1 in that a feature of individual differences in WM is the ability to flexibly allocate attention to items at encoding (i.e., filtering) and to select relevant mnemonic representations during maintenance intervals. Whereas Experiment 1 provided a spatial cue indicating which particular item would be tested, the cues in Experiment 2 were categorical, indicating the color of the to-be-remembered item. So Experiments 1 and 2 had several crucial differences. Experiment 1 tested participants' ability to allocate attention to one item using a spatial cue. In Experiment 2, the cue told participants which subset of the array would be tested. So rather than cueing to one specific item, the task cued participants to a subset of half the items. Second, in Experiment 2, the cue was categorical rather than spatial in nature. Third, the set size in Experiment 2 was larger to keep participants off ceiling and preserve between-subject variability. Importantly, Experiment 2 showed that WM span and attention control accounted for a significant portion of variance in precue trial performance, even after controlling for shared variance between neutral and precue trial performance. Further, while neutral trial performance and WM span accounted for variation in retrocue trial performance, attention control did not. These results indicate that there are several factors at work in determining how effectively WM can operate: selective encoding of relevant items, the ability to maintain a relatively large number of items, and the ability to select relevant items within memory for goal-directed behavior. 


\section{General discussion}

The present investigation had three aims: (1) to understand individual differences in the use of precues and retrocues in a visual WM task, (2) to examine how this relationship changed (or remained stable) as a function of cue type (i.e., spatial cues vs. categorical cues), and (3) to further delineate the relationships between attention control, capacity, and individual differences WM. In Experiment 1, we used a spatial cueing paradigm embedded in a visual WM task, and we measured more domain-general WM span with complex span tasks. WM span accounted for a small portion of variance in retrocues over and above the shared variance between neutral and cued trials performance. Admittedly, these relationships were a bit weak. As mentioned, performance on precue trials was quite high, and this may have reduced variability across participants.

In Experiment 2, we sought to extend Experiment 1 in several ways. First, we wanted to make the task a bit more difficult to create more variability across participants. We did so by using a larger set size and cueing to a subset of items rather than one specific item. Second, we wanted to use a categorical cue rather than a spatial cue. Third, we wanted to measure attention control abilities more directly. Neutral trial performance, WM span, and attention control all accounted for significant portions of variance in precue trial performance. This suggests that performance in precue trials was influenced by at least two abilities: (1) the ability to maintain several items in WM (i.e., capacity) and (2) the ability to selectively encode only relevant items (i.e., control). These findings are consistent with the idea that when WM requires filtering of irrelevant information, individuals need to exert attention to select and maintain only relevant information, and individuals with better attention control show an increased ability to do so beyond associated WM storage capacity advantages. The fact that WM span explained a significant portion of variance in precue trials even after controlling for variance due to neutral trial accuracy and attention control is an interesting finding. Typically, after controlling for attention control and short-term memory capacity, WM span differences are attributed to long-term memory retrieval (Unsworth \& Spillers, 2010). But it is not entirely clear whether the precue trials in Experiment 2 would require any memory retrieval abilities. Presumably, participants maintained the relevant set of information in an active state. It is possible that the test required participants to retrieve the tested item from that active set, which would reflect retrieval abilities. Another possibility is that the shared variance between precue trial performance and attention control reflects more general goal-maintenance abilities, like resisting mind-wandering. Although the thoughtsampling probes in the attention control tasks were not included for the present investigation specifically, we had those data. Averaging off-task (external distraction, mind-wandering, and mind-blanking) reports from the antisaccade and psychomotor vigilance tasks revealed a significant negative correlation between attention control and off-task thoughts $(r=-.51, p<$ $.001)$. However, controlling for off-task thoughts did not mediate the relationship between precue trials and attention control (partial $r=.34, p<.001$ ). So attention control was clearly playing a role other than reducing mindwandering and other off-task thoughts. A third possibility is that WM span is accounting for variance in the ability to dynamically update the task goal on every trial (i.e., remember red or remember blue). This would be consistent with goal-maintenance accounts of WM span (Kane \& Engle, 2003; Engle \& Kane, 2004). However, it is still not entirely clear what residual variance in precue performance WM span accounted for beyond capacity limitations and attention control abilities. So this finding deserves future research.

With respect to retrocue trials, WM span accounted for a significant portion of variance independently of shared variance with neutral trial accuracy, but attention control did not. We interpret this pattern of findings as follows: the ability to use retrocues to guide mnemonic representations requires at least two abilities: (1) the ability to maintain several items at once, and (2) the ability to act upon and retrieve relevant information once cued. Although attention control was positively correlated with retrocue trial performance, this relationship was mediated by neutral trial performance and WM span.

Our analyses relied heavily on multiple regression, and our logic closely followed that of Engle et al. (1999). Engle et al. argued that short-term memory and complex span WM share variance, and this shared variance reflects memory capacity. Further, they demonstrated that the WM residual (i.e., attention control), and the common variance between short-term memory and WM (i.e., memory capacity) each made independent contributions to fluid intelligence. In Experiment 1, our logic was as follows. When entered into a simultaneous regression predicting precue and retrocue trial performance, the shared variance between neutral trial performance and cued trial performance would primarily index differences in WM capacity, whereas any additional variance explained by WM span would primarily index attention control abilities. We strengthened this assumption in Experiment 2 by measuring attention control with two additional tasks. We do not argue that neutral trial performance is a process-pure measure of visual WM storage capacity, as almost no measure is process-pure. Further, we know that visual WM tasks without any sort of cue correlate with measures of attention control (Shipstead et al., 2015; Unsworth et al., 2014). But we argue that when entered into a simultaneous regression with WM span and attention control, the shared variance between neutral trials and cued trials captures any task-specific 
elements of capacity and control, whereas the independent WM span and attention control measures tap those constructs more generally.

We should also note that we do not argue that all forms of attention control are isomorphic. Indeed, control can take on a number of forms. During every task, a certain degree of goal maintenance is required. The task goal is to encode, maintain, and then make decisions about items in WM. Participants must continuously execute this goal. Further, participants must avoid diversions of attention away from the task goal (e.g., mind-wandering, external distraction). We and others have demonstrated that the ability to resist attentional failures on a trial-to-trial basis is a significant predictor of WM abilities (Adam, Mance, Fukuda, \& Vogel, 2015; Unsworth \& Robison, 2016). During precue trials, participants must select the item or subset of items that will be tested and ignore irrelevant items. During retrocue trials, participants must encode all items and then select the relevant item or subset of items during the retention interval. These are likely distinct yet related forms of control, and we argue they are all important determinants of individual differences in WM.

Overall, the findings indicate that in addition to being able to hold more items, as indexed by WM capacity, another aspect of individual differences in WM is the ability to flexibly allocate attention to items at encoding, and subsequently use memory retrieval abilities to select and act upon relevant representations. The findings are inconsistent with a pure capacity account, but they are also inconsistent with a pure attention control account. Just as with Mall et al. (2014), we did not observe significant interactions between the main effects of trial type and WM span. However, using regression techniques we examined shared and unique variance accounted for by capacity and the more attention control-specific aspects of WM span. In Experiment 2, we specifically measured attention control using two independent tasks, and we again found that WM span and control each accounted for significant amounts of variance in precue performance. These findings support a view that visual WM differences are a combination of storage capacity and attention control abilities. Finally, the fact that WM span accounted for a significant amount of variance in retrocue performance, but attention control did not, indicates that the use of retrocues may require WM span-related retrieval abilities. This makes sense given the nature of the task. Individuals must first maintain a large set of items, in this case six, and then must use a retrocue to select a relevant subset of those items. Finally, the individual has to make a judgment about a particular item based on the mnemonic representation. This may require certain degree of retrieval, as visual shortterm memory capacity is pushed to its limit.

As mentioned earlier, there is considerable debate as to how exactly retrocues operate to improve WM performance. To distinguish between the various mechanisms, experiments have manipulated array-cue delays, cue-test delays, number of cues, set sizes, and cue validity. In the present set of experiments, we included only one within-subject manipulation: trial type (precue, neutral, and retrocue). The primary purpose of the present study was to examine individual differences in the use of cues and their relation to visual WM capacity, WM span, and attention control. Therefore, we tried to keep as many of the above-mentioned manipulations as constant as possible. We chose to use a set size at or near the capacity of the majority of participants. Using smaller set sizes may have made rendered the cues obsolete, as participants would have been able to encode and maintain all items, regardless of the cue. Similarly, the use of larger set sizes may have simply made the cues more helpful for high-WM participants because they would have had a higher likelihood of cueing an actively maintained item. Therefore we decided to keep set size constant within each experiment. Additionally we wanted to encourage the use of the cues to maximize performance, so we did not include invalidly cued trials. Past experiments have shown that invalidly cued trials may lead some participants to ignore the cues, or at least reduce the retrocue benefit (Berryhill et al., 2012; Gözenman et al., 2014; Matsukura et al., 2007; Williams \& Woodman, 2012). Especially for high-WM individuals, invalid trials may encourage participants to try to maintain all items rather than face the cost of using an invalid cue. For that reason we did not include invalid-cue trials. Finally, because we were not specifically interested in the temporal effects of the cues, we kept the cue-array, array-cue, array-test, and cue-test delays constant within each experiment. Although this may have led to some expectancies within participants, in both experiments trial types were randomly intermixed. Therefore, because participants did not know the trial type, we do not believe the fixed delays systematically alter our interpretations of the results. As a result of fixing many of these experimental parameters, it is difficult for us to speculate as to how variation in WM capacity and attention control relate to the various theoretical mechanisms behind retrocue benefits. Future iterations of the present design with specific task manipulations will be necessary to distinguish among the various possibilities. However, it is clear that high-WM participants are utilizing their attention to select and maintain items in visual WM more effectively than their low-WM counterparts.

\section{Conclusion}

Although there has long been an association between attention control and WM capacity, the precise nature of this relationship within visual WM is still not entirely understood. Some have argued that individual differences in visual WM largely reflect the ability to gate access to WM. Other theories have 
argued that WM is largely due to the amount of information an individual can hold active in mind. Within visual WM tasks, there is some evidence to suggest that attention control plays a critical role in determining individual differences in WM (Fukuda \& Vogel, 2009, 2011; Vogel et al., 2005), but recent investigations have challenged this view (Mall et al., 2014). Therefore, ongoing research must continue to investigate this relationship. In the present set of experiments, the evidence suggests that in addition to being able to maintain a greater number of items in WM (i.e., capacity), an element of individual differences in WM is the ability to adjust attention to items in two ways: the selective encoding of relevant information and the prioritized maintenance of relevant information. This finding was not specific to spatial attention, but extended to a more feature-based, categorical allocation of attention. Thus we argue that the present results support the view that visual WM is not simply a measurement of the amount of information that can be maintained in an active state, nor simply attention control, but rather it is a complex, multiply determined cognitive ability that has aspects of capacity and control (Cowan et al., 2006; Engle et al., 1999; Shipstead et al., 2012, 2015; Unsworth et al., 2014; Unsworth, 2016).

\section{References}

Adam, K. S., Mance, I., Fukuda, K., \& Vogel, E. K. (2015). The contribution of attentional lapses to individual differences in visual working memory capacity. Journal of Cognitive Neuroscience, 27, 16011616.

Astle, D. E., Nobre, A. C., \& Scerif, G. (2012). Attentional control constrains visual short-term memory: Insights from developmental and individual differences. The Quarterly Journal of Experimental Psychology, 65, 277-294.

Astle, D. E., \& Scerif, G. (2011). Interactions between sttention and visual short-term memory (VSTM): What can be learnt from individual and developmental differences? Neuropsychologia, 49, 1435-1445.

Astle, D. E., Scerif, G., Kuo, B.-C., \& Nobre, A. C. (2009). Spatial selection of features within perceived and remembered objects. Frontiers in Human Neuroscience, 3, 6.

Astle, D. E., Summerfield, J., Griffin, I., \& Nobre, A. C. (2012). Orienting attention to locations in mental representations. Attention, Perception, \& Psychophysics, 74, 146-162.

Awh, E., Vogel, E. K., \& Oh, S.-H. (2006). Interactions between attention and working memory. Neuroscience, 139, 201-208.

Berryhill, M. E., Richmond, L. L., Shay, C. S., \& Olson, I. R. (2012). Shifting attention among working memory representations: Testing cue type, awareness, and strategic control. The Quarterly Journal of Experimental Psychology, 65, 426-438.

Chuderski, A., \& Necka, E. (2012). The contribution of working memory to fluid reasoning: Capacity, control, or both? Journal of Experimental Psychology: Learning, Memory, and Cognition, 38, $1689-1710$

Chun, M. M., \& Johnson, M. K. (2011). Memory: Enduring traces of perceptual and reflective attention. Neuron, 72, 520-535.

Cowan, N., Elliott, E. M., Saults, J. S., Morey, C. C., Mattox, S., Hismjatullina, A., \& Conway, A. R. A. (2005). On the capacity of attention: Its estimation and its role in working memory and cognitive aptitudes. Cognitive Psychology, 51, 42-100.

Cowan, N., Fristoe, N. M., Elliott, E. M., Brunner, R. P., \& Saults, J. S. (2006). Scope of attention, control of attention, and intelligence in children and adults. Memory \& Cognition, 34, 1754-1768.

Cowan, N., \& Morey, C. C. (2006). Visual working memory depends on attentional filtering. Trends in Cognitive Sciences, 10, 139-141.

Dinges, D. F., \& Powell, J. W. (1985). Microcomputer analysis of performance on a portable, simple visual RT task during sustained operations. Behavior Research Methods, Instruments and Computers, 17, 652-655.

Duarte, A., Hearons, P., Jiang, Y., Delvin, M. C., Newsome, R. N., \& Verhaeghen, P. (2013). Retrospective attention enhances visual working memory in the young but not the old. Psychophysiology, 50, 465-476.

Engle, R. W., \& Kane, M. J. (2004). Executive attention, working memory capacity, and a two- factor theory of cognitive control. In B. H. Ross (Ed.), The psychology of learning and motivation (Vol. 44, pp. 145-199). New York: Academic Press.

Engle, R. W., Tuholski, S. J., Laughlin, J. E., \& Conway, A. R. A. (1999). Working memory, short-term memory, and general fluid intelligence: A latent variable approach. Journal of Experimental Psychology: General, 128, 309-331.

Fukuda, K., \& Vogel, E. K. (2009). Human variation in overriding attentional capture. The Journal of Neuroscience, 29, 8726-8733.

Fukuda, K., \& Vogel, E. K. (2011). Individual differences in recovery time from attention capture. Psychological Science, 22, 361-368.

Gilchrist, A. L., Duarte, A., \& Verhaeghen, P. (2016). Retrospective cues based on object features improve visual working memory performance in older adults. Aging, Neuropsychology, and Cognition, 23, 184-195.

Gözenman, F., Tanoue, R. T., Metoyer, T., \& Berryhill, M. E. (2014). Invalid retro-cues can eliminate the retro-cue benefit: evidence for a hybridized account. Journal of Experimental Psychology: Human Perception and Performance, 40, 1748-1754.

Griffin, I. C., \& Nobre, A. C. (2003). Orienting attention to locations in internal representations. Journal of Cognitive Neuroscience, 15, 1176-1194.

Kane, M. J., Bleckley, M. K., Conway, A. R. A., \& Engle, R. W. (2001). A controlled-attention view of working-memory capacity. Journal of Experimental Psychology: General, 130, 169-183.

Kane, M. J., \& Engle, R. W. (2003). Working-memory capacity and the control of attention: The contributions of goal maintenance, response competition, and task set to Stroop interference. Journal of Experimental Psychology: General, 132, 47-70.

Kane, M. J., Meier, M. E., Smeekens, B. A., Gross, G. M., Chun, C. A., Silvia, P. J., \& Kwapil, T. R. (2016). Individual differences in executive control of attention, memory, and thought, and their associations with schizotypy. Journal of Experimental Psychology: General, 145, 1017-1048.

Kline, R. B. (2011). Principles and practice of structural equation modeling. New York: Guilford Press.

Landman, R., Spekreijse, H., \& Lamme, V. A. F. (2003). Large capacity storage of integrated objects before change blindness. Vision Research, 43, 149-164.

Lepsien, J., \& Nobre, A. C. (2007). Attentional modulation of object representations in working memory. Cerebral Cortex, 17, 2072 2083.

Makovski, T., \& Jiang, Y. V. (2007). Distributing versus focusing attention in visual short-term memory. Psychonomic Bulletin \& Review, 14, 1072-1078.

Makovski, T., Sussman, R., \& Jiang, Y. V. (2008). Orienting attention in visual working memory reduces interference from memory probes. Journal of Experimental Psychology: Learning, Memory, and Cognition, 34, 369-380. 
Mall, J. T., Morey, C. C., Wolff, M. J., \& Lehnert, F. (2014). Visual selective attention is equally functional for individuals with low and high working memory capacity: Evidence from accuracy and eye movements. Attention, Perception, \& Psychophysics, 76, 1998-2014.

Matsukura, M., Luck, S. J., \& Vecera, S. P. (2007). Attention effects during visual short-term memory maintenance: Protection or prioritization? Perception \& Psychophysics, 69, 1422-1434.

McVay, J. C., \& Kane, M. J. (2012). Drifting from slow to "D'oh!": Working memory capacity and mind wandering predict extreme reaction times and executive-control errors. Journal of Experimental Psychology: Learning, Memory, and Cognition, 38, $525-549$.

Mok, R. M., Myers, N. E., Wallis, G., \& Nobre, A. C. (2016). Behavioral and neural markers of flexible attention over working memory in aging. Cerebral Cortex, 26, 1831-1842.

Pertzov, Y., Bays, P. M., Joseph, S., \& Husain, B. (2013). Rapid forgetting prevented by retrospective attention cues. Journal of Experimental Psychology: Human Perception and Performance, 39, 1224-1231.

Redick, T. S., Shipstead, Z., Meier, M. E., Montroy, J. J., Hicks, K. L., Unsworth, N., ... Engle, R. W. (2016). Cognitive predictors of a common multitasking ability: Contributions from working memory, attention control, and fluid intelligence. Journal of Experimental Psychology: General, 145, 1473-1492.

Robison, M. K., \& Unsworth, N. (2017a). Individual differences in working memory capacity and resistance to belief bias in syllogistic reasoning. The Quarterly Journal of Experimental Psychology, 70, 1471-1484.

Robison, M. K., \& Unsworth, N. (2017b). Working memory capacity, strategic allocation of study time, and value-directed remembering. Journal of Memory and Language, 93, 231-244.

Shimi, A., Kuo, B.-C., Astle, D. E., Nobre, A. C., \& Scerif, G. (2014). Age group and individual differences in attentional orienting dissociate neural mechanisms of encoding and maintenance in visual STM. Journal of Cognitive Neuroscience, 26, 864-877.

Shipstead, Z., Harrison, T. L., \& Engle, R. W. (2015). Working memory capacity and the scope and control of attention. Attention, Perception, and Psychophysics, 77, 1863-1880.

Shipstead, Z., Redick, T. S., Hicks, K. L., \& Engle, R. W. (2012). The scope and control of attention as separate aspects of working memory. Memory, 20, 608-628.

Slitge, I. G., Scholte, H. S., \& Lamme, V. A. F. (2008). Are there multiple visual short-term stores? PLOS ONE, 3, e1699.

Souza, A. S., \& Oberauer, K. (2016). In search of the focus of attention in working memory: 13 years of the retrocue effect. Attention, Perception, \& Psychophysics, 78, 1839-1860.

Souza, A. S., Rerko, L., \& Oberauer, K. (2016). Getting more from visual working memory: Retro-cues enhance retrieval and protect from visual interference. Journal of Experimental Psychology: Human Perception and Performance, 42, 890-910.

Sperling, G. (1960). The information available in brief visual representations. Psychological Monographs: General and Applied, 74, 1-19.

Unsworth, N. (2016). The many facets of individual differences in working memory capacity. In B. Ross (Ed.).The psychology of learning and motivation (Vol. 65, pp. 1-46).

Unsworth, N., Brewer, G. A., \& Spillers, G. J. (2012). Variation in cognitive failures: An individual differences investigation of everyday attention and memory failures. Journal of Memory and Language, 67, 1-16.

Unsworth, N., \& Engle, R. W. (2007). The nature of individual differences in working memory capacity: Active maintenance in primary memory and controlled search from secondary memory. Psychological Review, 114, 104-132.

Unsworth, N., Fukuda, K., Awh, E., \& Vogel, E. K. (2014). Working memory and fluid intelligence: Capacity, attention control, and secondary memory. Cognitive Psychology, 71, 1-26.

Unsworth, N., Fukuda, K., Awh, E., \& Vogel, E. K. (2015). Working memory delay activity predicts individual differences in cognitive abilities. Journal of Cognitive Neuroscience, 27, 853-865.

Unsworth, N., \& McMillan, B. D. (2014). Similarities and differences between mind-wandering and distraction A latent variable analysis of lapses of attention and their relation to cognitive abilities. Acta Psychologica, 150, 14-25.

Unsworth, N., McMillan, B. D., Brewer, G. A., \& Spillers, G. J. (2012). Everyday attention failures: An individual differences investigation. Journal of Experimental Psychology: Learning, Memory, \& Cognition, 38, 1765-1772.

Unsworth, N., \& Robison, M. K. (2015). Individual differences in the allocation of attention to items in working memory: Evidence from pupillometry. Psychonomic Bulletin \& Review, 22, 757-765.

Unsworth, N., \& Robison, M. K. (2016). The influence of lapses of attention on working memory capacity. Memory \& Cognition, 44, 188-196.

Unsworth, N., \& Spillers, G. J. (2010). Working memory capacity: Attention control, secondary memory, or both? A direct test of the dual-component model. Journal of Memory and Language, 62, 392-406.

Vogel, E. K., McCollough, A. W., \& Machizawa, M. G. (2005). Neural measures reveal individual differences in controlling access to working memory. Nature, 438, 500-503.

Williams, M., Hong, S. W., Kang, M.-S., Carlisle, N. B., \& Woodman, G. F. (2013). The benefit of forgetting. Psychonomic Bulletin \& Review, 20, 348-355.

Williams, M., \& Woodman, G. F. (2012). Directed forgetting and directed remembering in visual working memory. Journal of Experimental Psychology: Learning, Memory, and Cognition, $38,1206-1220$. 